\title{
Cartilage oligomeric matrix protein in patients with osteoarthritis is independently associated with metastatic disease in prostate cancer
}

\author{
Samuel Rosas ${ }^{1,2}$, Ryan T. Hughes', Michael Farris', Hwajin Lee', Emory R. \\ McTyre $^{3}$, Johannes F. Plate ${ }^{2}$, Lihong Shi ${ }^{4}$, Cynthia L. Emory ${ }^{2}$, A. William Blackstock ${ }^{1}$ \\ Bethany A. Kerr ${ }^{2,4}$ and Jeffrey S. Willey ${ }^{1,2}$ \\ ${ }^{1}$ Department of Radiation Oncology, Wake Forest School of Medicine, Winston-Salem, NC, USA \\ 2 Department of Orthopaedic Surgery, Wake Forest School of Medicine, Winston-Salem, NC, USA \\ ${ }^{3}$ Radiation Oncology, Greenville Health System Cancer Institute, Greenville, SC, USA \\ ${ }^{4}$ Department of Cancer Biology, Wake Forest School of Medicine, Winston-Salem, NC, USA \\ Correspondence to: Jeffrey S.Willey, email: jwilley@wakehealth.edu \\ Keywords: prostate cancer; osteoarthritis; distant metastasis; arthroplasty; joint surgery \\ Received: April 16, $2019 \quad$ Accepted: June 29, 2019, Published: July 30, 2019 \\ Copyright: Rosas et al. This is an open-access article distributed under the terms of the Creative Commons Attribution License 3.0 \\ (CC BY 3.0), which permits unrestricted use, distribution, and reproduction in any medium, provided the original author and source \\ are credited.
}

\section{ABSTRACT}

Metastatic prostate cancer has a 5-year survival rate of $30 \%$. Identifying predictors of metastasis outcome could potentially reduce patient mortality. The objective of this study was to determine whether osteoarthritis had an impact on outcomes of prostate cancer including death, local recurrence and/or metastasis and to determine whether cartilage oligomeric matrix protein was involved. We performed a retrospective case-control study of patients with prostate cancer with and without the diagnosis of osteoarthritis and completed immunohistochemistry (IHC) analysis of prostate $(n=20)$ and lymph node $(n=7)$ surgical specimens. We evaluated death, local recurrence and metastatic disease by various IHC biomarkers including prostate specific membrane antigen (PSMA), cartilage oligomeric matrix protein (COMP), CD31, and $\mathrm{Ki}-67$.

Our model identified osteoarthritis as an independent risk factor for metastatic disease (OR 5.24, 95\% CI 1.49 - 18.41). Most notably, when joint arthroplasty was included in the model, osteoarthritis was no longer an independent risk factor for this outcome $(p=0.071)$. IHC demonstrated that those with osteoarthritis, had greater expression of COMP in the prostate samples (mean $23.9 \%$ vs $5.84 \%, p<0.05$ ) but not of Ki-67, CD31, or PSMA. This study identified and quantified increased metastatic disease in patients with osteoarthritis. Also, patients with osteoarthritis expressed increased COMP levels in the prostate and most likely in distant lymphatic nodes. Moreover, our findings suggest that joint arthroplasty may affect the ability of osteoarthritis to promote metastasis, which could impact treatment protocols and survival outcomes of the most common cause of cancer-related death (metastasis) in the United States.

\section{INTRODUCTION}

Prostate cancer is the second most common cause of cancer-related deaths in American men [1]. Primary treatment includes androgen deprivation therapy, radiation, or radical prostatectomy [2], and despite recent advances in systemic and local treatment, for patients with metastatic disease survival outcomes remain poor $[3,4]$. Moreover, the economic effects of this disease on the U.S. healthcare system are considerable with some authors 
Table 1: Demographic and patient characteristics based on osteoarthritis

\begin{tabular}{lccc}
\hline \multicolumn{1}{c}{$\begin{array}{c}\text { Demographic Data } \\
\text { Stratified by OA }\end{array}$} & $\begin{array}{c}\text { Osteoarthritis } \\
(\mathbf{n}=\mathbf{7 8})\end{array}$ & $\begin{array}{c}\text { No Osteoarthritis } \\
(\mathbf{n = 1 9 6})\end{array}$ & p value \\
\hline $\begin{array}{l}\text { Mean Age in Years at } \\
\text { Diagnosis (SD) }\end{array}$ & $66.9(7.9)$ & $65.9(8.3)$ & 0.383 \\
Mean Follow Up Months & $60.8(87.99)$ & $85.3(107.4)$ & 0.332 \\
Median (IQR) & & & \\
Gleason Score (\%) & 26.9 & 44.9 & 0.016 \\
$\quad 6$ or below & 35.9 & 32.7 & \\
$\quad 7$ & 34.6 & 21.9 & 1 \\
$\quad 8$ or higher & $100 \%$ & $100 \%$ & 0.064 \\
Radiotherapy & $7.70 \%$ & $4.10 \%$ & 0.015 \\
Chemotherapy & $58.5 \%$ & $41.5 \%$ & 0.531 \\
Hormonal Therapy & $11.70 \%$ & $9.70 \%$ & \\
Treatment with Surgery & &
\end{tabular}

estimating total expenditure at over 9.8 billion dollars in 2006 [5]. Recent data by Trogdon et al. revealed a 1.2 billion dollar cost for elderly men with prostate cancer in only 3 years [6]. Significant effort has been directed at identifying causes, complications, and risk factors for prostate cancer progression in order to improve survival and decrease the cost burden. However, there may be patient comorbidities that play a role in promoting disease progression and have yet to be identified [7, 8].

Recent translational research described a novel pathway that stimulated prostate cancer progression in vivo and in vitro including Cartilage Oligomeric Matrix Protein (COMP); a small (54 kD) molecule most commonly found in the extracellular matrix (ECM) of cartilage $[9,10]$. Englund et al. described that COMP promoted

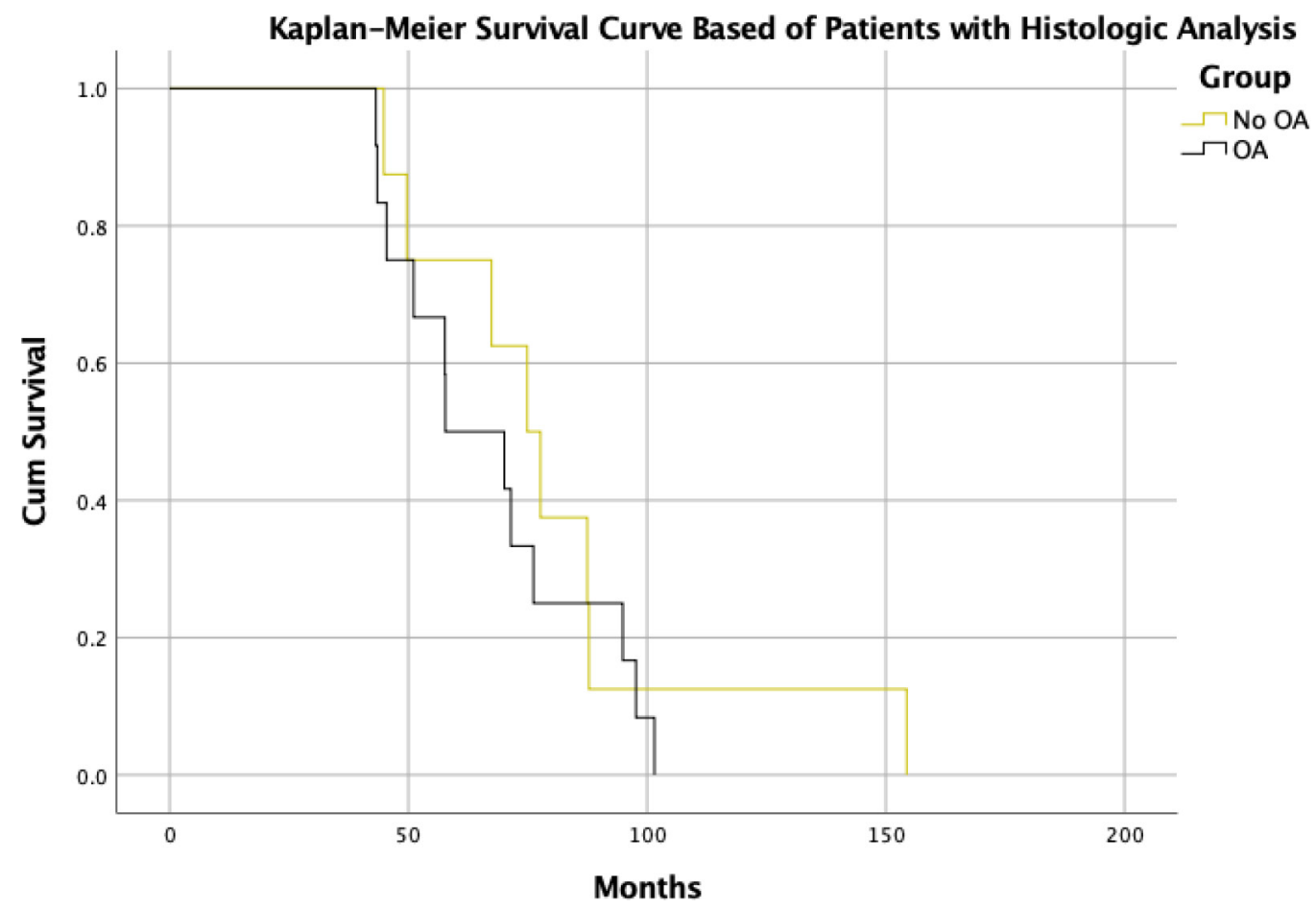

Figure 1: Kaplan-Meier Survival curve of patients with clinical and histological data. 
Table 2: Clinical Outcomes

\begin{tabular}{lccc}
\hline $\begin{array}{c}\text { Outcomes of } \\
\text { Prostate Cancer }\end{array}$ & $\begin{array}{c}\text { Osteoarthritis } \\
(\mathbf{n = 7 8})\end{array}$ & $\begin{array}{c}\text { No Osteoarthritis } \\
(\mathbf{n = 1 9 6})\end{array}$ & p value \\
\hline $\begin{array}{l}\text { Overall Survival } \\
\text { (SD) in Months }\end{array}$ & $81.6(53.9)$ & $89.1(114.7)$ & 0.600 \\
Local Recurrence & $1.50 \%$ & $0.40 \%$ & 0.878 \\
Metastasis & $3 \%$ & $1.80 \%$ & 0.003 \\
\hline
\end{tabular}

progression by stimulating tumor invasion due to changes in intracellular calcium release from the endoplasmic reticulum and in oxidative phosphorylation [9]. The authors demonstrated the presence of COMP in $16 \%$ of tumor tissue microarrays and found a significant association with COMP expression and time to metastases and to biochemical recurrence (defined as a PSA $>0.2 \mathrm{ng} / \mathrm{ml}$ ).
COMP also functions as an ECM stabilizer, and is upregulated with joint loading during exercise and secreted to the serum in patients with osteoarthritis [11]. However, the effects of COMP on prostate cancer progression in patients with osteoarthritis have not been studied and could present a novel therapeutic approach to prostate cancer treatment. The purpose of this study was to evaluate whether osteoarthritis was an independent risk factor for adverse
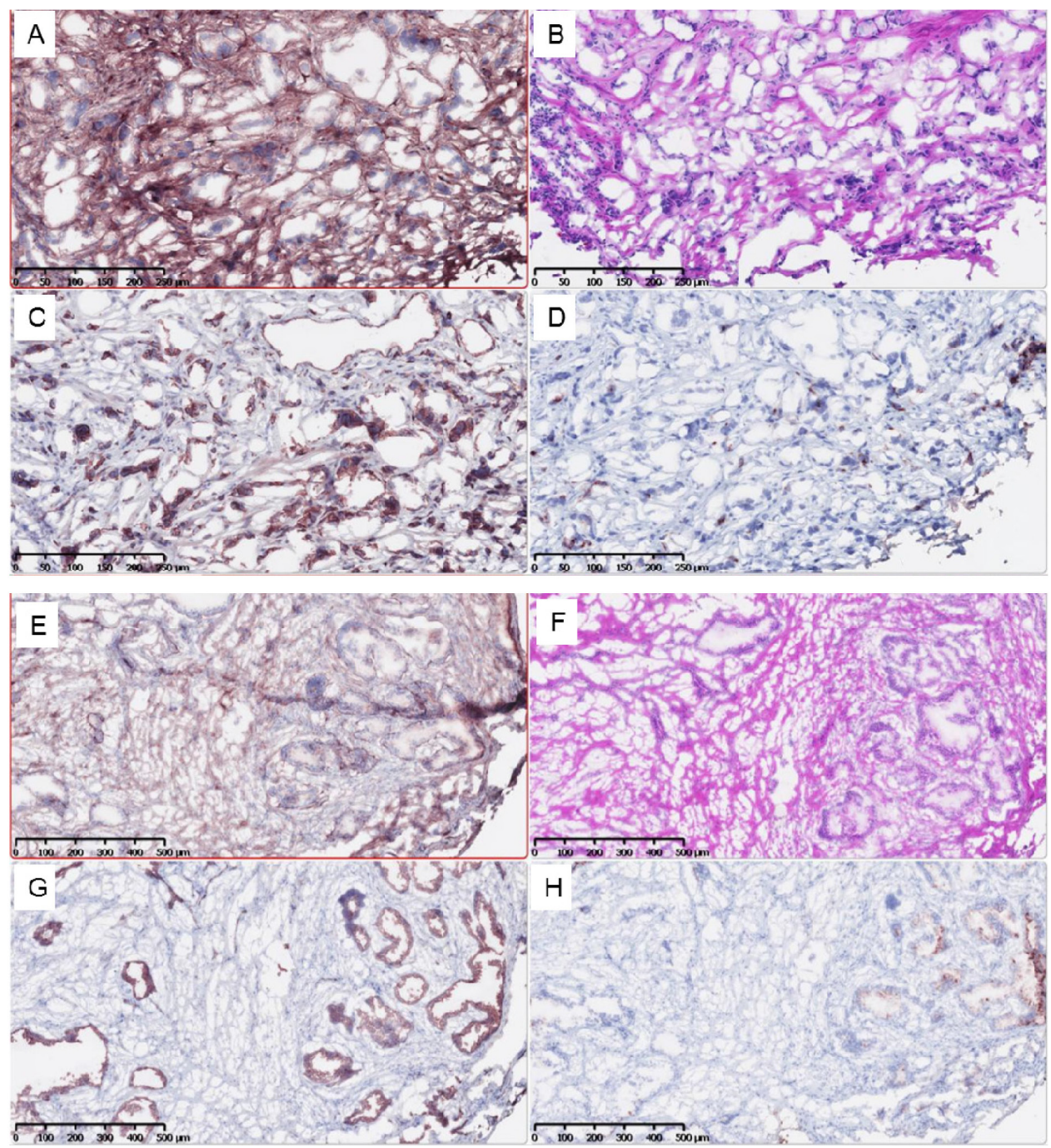

Figure 2: Lymph Nodes of Patient with osteoarthritis (top 4 images) and no osteoarthritis (bottom). (A,E) PSMA; (B,F) H\&E, (C,G) Ki-67, (D,H) COMP. 
Table 3: Logistic regression model predictive of mortality

\begin{tabular}{lcccc}
\hline \multicolumn{1}{c}{ Risk Factor } & Odds Ratio & \multicolumn{2}{c}{ 95\% Confidence Interval } & p value \\
\hline Osteoarthritis & 0.815 & 0.405 & 1.641 & 0.567 \\
Age at diagnosis & 1.059 & 1.018 & 1.100 & 0.004 \\
Gleason Score & 1.004 & 0.717 & 1.407 & 0.981 \\
Chemotherapy & 13.485 & 3.610 & 50.376 & $<0.001$ \\
Hormone Therapy & 0.539 & 0.242 & 1.201 & 0.131 \\
Prostatectomy & 0.731 & 0.241 & 2.222 & 0.581 \\
Constant & 0.007 & & & .002 \\
\hline
\end{tabular}

outcomes of prostate cancer. Our secondary aim was to determine whether a diagnosis of osteoarthritis correlated with upregulation of tumor markers associated with angiogenesis (CD31), proliferation (Ki-67) and the presence of COMP. The study hypothesized that osteoarthritis is an independent risk factor for worse outcomes of prostate cancer patients as it pertains to overall survival, local recurrence, and metastasis while accounting for other comorbidities [9, 12]. The findings of this study could lead to a change in the standard of care for patients with osteoarthritis and prostate cancer and the potential value that orthopedic surgery could provide for these patients.

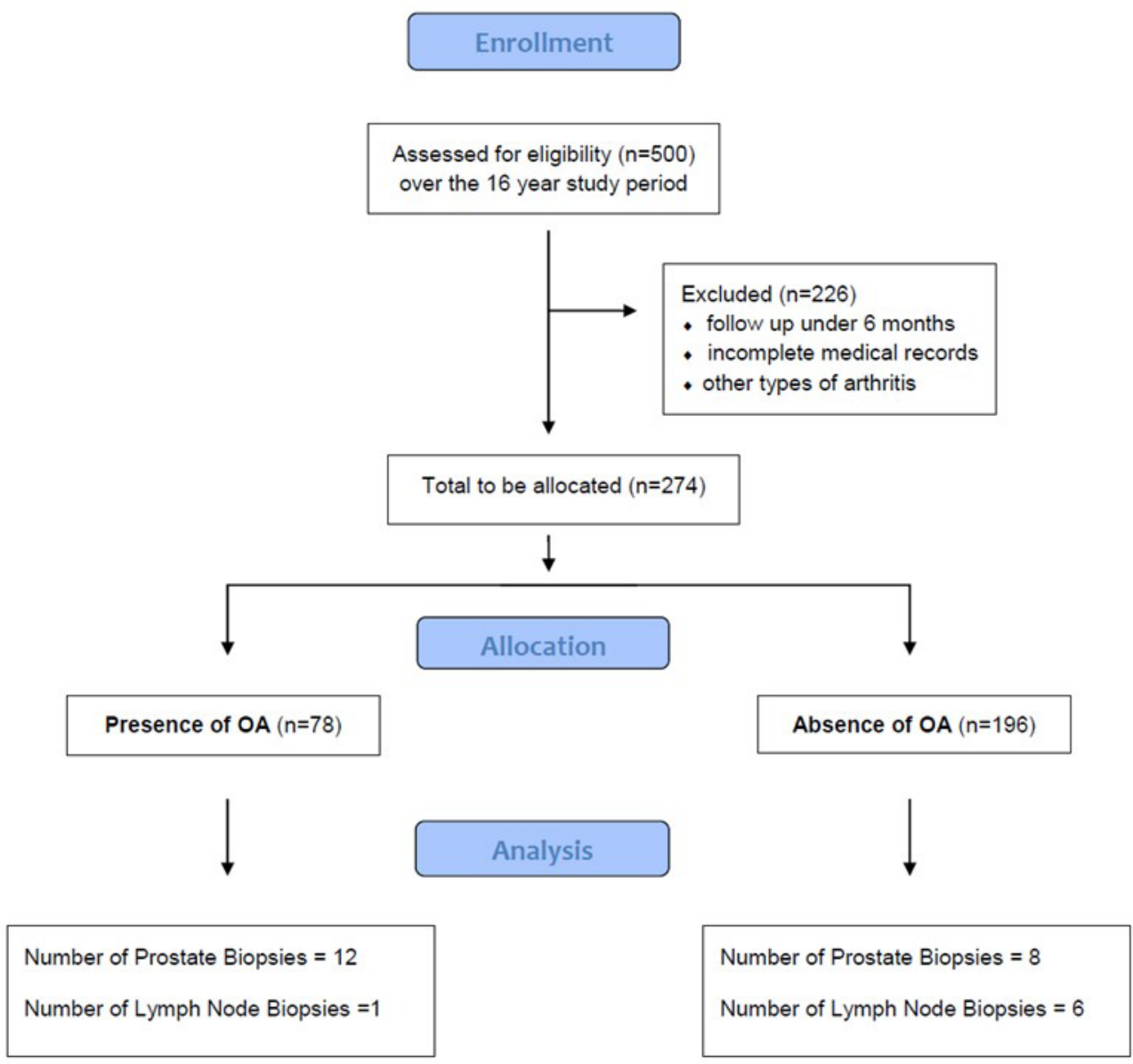

Figure 3: Consort Flow diagram of study design. 
Table 4: Logistic regression model predictive of metastasis

\begin{tabular}{lcccc}
\hline \multicolumn{1}{c}{ Risk Factor } & Odds Ratio & 95\% Confidence Interval & p value \\
\hline Osteoarthritis & 5.244 & 1.493 & 18.414 & 0.01 \\
Age at diagnosis & 0.882 & 0.811 & 0.958 & 0.003 \\
Gleason Score & 0.261 & 0.178 & 1.172 & 0.103 \\
Prostatectomy & 4.557 & 1.144 & 18.160 & 0.032 \\
Local Recurrence & 10.057 & 0.351 & 288.349 & 0.178 \\
Constant & .4708 & & & 0.630 \\
\hline
\end{tabular}

\section{RESULTS}

A total of 274 patients with a mean age at prostate cancer diagnosis of 77.1 years (SD 9.33) were included in the study. The overall mean follow-up was 79.5 months (range 6.6-201.26). Survival of the entire cohort was $77 \%$ at last follow up. There were 78 patients $(28 \%)$ with a concomitant diagnosis of osteoarthritis and 196 patients $(72 \%)$ without osteoarthritis. Table 1 highlights the characteristics of the studied population as stratified by osteoarthritis presence. Of the 78 patients with osteoarthritis, $10.2 \%$ underwent joint arthroplasty.

There were no differences in patient demographics between the osteoarthritis and no osteoarthritis groups (Table 2). However, patients with osteoarthritis had a significantly higher Gleason score at the time of diagnosis compared to patients without osteoarthritis (Table 2). While overall survival was similar between groups, patients with osteoarthritis did had a significantly higher rate of metastatic disease during follow up compared to those without OA, $3 \%$ vs $1.8 \%(p=0.003)$.

\section{Osteoarthritis effects on mortality}

In the logistic regression model evaluating independent predictors of mortality, only age at diagnosis $(p=0.004)$ and use of chemotherapy $(p<0.001)$ were independent predictors of death (Table 3). Survival time was similar with and without osteoarthritis while accounting for covariates $(\mathrm{p}=0.915)$. However, there was a numerical improvement in survival in OA patients who received joint arthroplasty that did not reach statistical significance $(\mathrm{p}=0.064)$.

\section{Osteoarthritis effects on local recurrence}

The model used to identify independent predictors of local recurrence was statistically significant $\left(\mathrm{X}^{2}: 21.53, \mathrm{p}=0.006\right)$ at predicting local recurrence rates. Although this model correctly classified $98.5 \%$ of cases of local recurrence, osteoarthritis was not found to be an independent predictor of local recurrence as well as any other predictor included $(\mathrm{p}>0.05)$.

\section{Osteoarthritis effects on distant metastasis}

The model used to identify independent predictors of metastasis was statistically significant $\left(\mathrm{X}^{2}: 47.04, \mathrm{p}<\right.$ 0.001 ) as it correctly classified $95.5 \%$ of patients who developed metastatic disease. There were four independent predictors of distant metastasis: age at diagnosis, surgical treatment, and a diagnosis of osteoarthritis $(\mathrm{p}<0.05)$. Table 4 demonstrates the odds ratios of each of the predictors. We then developed a model with the same predictors including joint arthroplasty, and chemotherapy and demonstrated similar statistical ability to discriminate metastasis $\left(\mathrm{X}^{2}: 45.47, \mathrm{p}<0.001\right)$ classifying $95.1 \%$ of cases correctly. Notably, this model identified similar predictors of distant recurrence (age at diagnosis, treatment with chemotherapy, and treatment with surgery) except for a diagnosis of osteoarthritis $(\mathrm{p}=0.071)$ as demonstrated in Table 5 .

\section{Histologic evaluation of prostate cancer samples}

A total of twenty samples were evaluated histologically in this study; eight were of patients with osteoarthritis and twelve of those without osteoarthritis (Table 6, Figure 1 and 2). A total of seven samples from lymphatic nodes were available for analysis. In the prostate samples, COMP expression was significantly greater in patients with osteoarthritis (mean $23.9 \%$ vs $5.85 \%, \mathrm{p}<0.05)$. The percent of positivity for CD31, $\mathrm{Ki}-67$, and PSMA was similar in both groups ( $\mathrm{p}>0.05$ for all). Table 6 demonstrates the IHC depiction of the samples and the characteristics of the patients studied. Due to the limited sample size available, only one was of a patient with osteoarthritis, which coincided with the patient who expressed the greatest amount of COMP $(61.3 \%)$, compared to $30.7 \%$ in the highest sample of patients without osteoarthritis (Table 7). The patient with osteoarthritis also presented with the highest Gleason score and developed metastatic disease (Figure 2).

\section{DISCUSSION}

The current study evaluated the influence of osteoarthritis and subsequent upregulation of COMP on 
Table 5: Modified Logistic regression model predictive of metastasis

\begin{tabular}{lcccc}
\hline \multicolumn{1}{c}{ Risk Factor } & Odds Ratio & $\mathbf{9 5 \%}$ Confidence Interval & p value \\
\hline Osteoarthritis & 4.213 & 0.885 & 20.062 & 0.071 \\
Age at diagnosis & 0.897 & 0.821 & 0.980 & 0.016 \\
Gleason Score & 1.057 & 0.540 & 2.070 & 0.872 \\
Chemotherapy Given & 36.390 & 5.637 & 234.923 & 0.000 \\
Prostatectomy & 4.905 & 1.060 & 22.690 & 0.042 \\
Joint Arthroplasty & 2.792 & 0.457 & 17.042 & 0.266 \\
Constant & 6.214 & & & 0.610 \\
\hline
\end{tabular}

the prostate cancer presentation and disease progression. Osteoarthritis was identified as an independent predictor of metastatic disease (OR 5.24, 95\% CI 1.49 - 18.41, $\mathrm{p}=0.01$ ). While the underlying pathophysiology remains to be elucidated, there may be similarities to rheumatoid arthritis [13, 14]. Although rheumatoid arthritis has a different pathophysiology from osteoarthritis, it leads to chronic joint damage that can stimulate COMP production and secretion into the blood stream [15]. Theoretically, joint injury leads to increased systemic COMP, which then stimulates tumor progression [15]. A registry based study of Medicare patients in Texas, correlated the presence of rheumatoid arthritis with increased mortality in prostate cancer patients by $50 \%$ after controlling for other comorbidities [14]. A more recent systematic review failed to identify rheumatoid arthritis as causing a greater risk of developing prostate cancer compared to the normal population [16] and these studies highlight the lack of knowledge on this topic. Our findings lead us to believe that osteoarthritis or rheumatoid arthritis could increase cancer proliferation but not cause tumor initiation, as has been demonstrated in vitro [9].

Patient survival was not influenced by osteoarthritis in this study. However, this finding may be confounded due to various factors. First, the disease usually progresses slowly with metastases developing after 10 years, and prostate cancer is often not the cause of death for patients, as death from cardiovascular disease predominates [17]. Second, despite a followup duration of over 6 years in the present cohort, the indolent nature of the prostate cancer may limit the power to detect a difference in overall survival. It may be possible that with longer follow-up, osteoarthritis may be associated with survival. Nonetheless, the survival rate of the patients in this cohort corresponding to findings from previous studies [17-19].

Osteoarthritis was not found to be an independent predictor of local cancer recurrence. This finding was not surprising as the likelihood of local recurrence has been more commonly correlated with pre-treatment PSA levels, Gleason scores, and various imaging parameters [20-22]. Moreover, local recurrence may be identified by biochemical recurrence, symptomatic disease, and/ or radiographic findings, making its definition in the literature somewhat variable and limiting the interpretation of this lack of independent predictor identification $[10,17$, 19-21].

An interesting finding that requires further study is that osteoarthritis was no longer an independent predictor of metastasis in patients who had undergone joint arthroplasty. While the underlying mechanism remains to be identified, this finding may have occurred due to selfselection of healthier patients undergoing arthroplasty for osteoarthritis versus surgeons not offering arthroplasty to patients with a high likelihood of unsatisfactory results (such as patients with advanced prostate cancer). Joint arthroplasty removes the areas of the joint involved in osteoarthritis and could thereby lead to decreased systemic levels of COMP, which in turn would reduce COMPmediated promotion of cancer proliferation compared to osteoarthritis patients who did not undergo arthroplasty. The trend toward increased survival time of prostate cancer patients with osteoarthritis after joint arthroplasty warrants further investigation.

The IHC analysis revealed that COMP levels were significantly higher among biopsies of patients with osteoarthritis, compared to patients without osteoarthritis. This finding may be due to dysregulation of the ECM, promoted by increased COMP in the serum that travels systemically which could promote greater metastatic disease and distant seeding of tumor cells. Nonetheless, this is the first report of this relationship, and future studies are needed to evaluate systemic levels of COMP and possible temporal changes in COMP expression to further examine the interaction between osteoarthritis and prostate cancer.

Regarding the equal expression of CD31, PSMA and $\mathrm{Ki}-67$ in both cohorts of patients, one could hypothesize that the greater metastatic disease seen in patients with osteoarthritis was not likely due to increased angiogenesis or direct tumor proliferation but due to cancer invasion into the adjacent tissues. Unfortunately, there are mixed opinions in the literature as for how these markers correlate with clinical tumor progression [23-25].

The observation of increased COMP expression in a lymph node of an osteoarthritis patient of twice the amount 
Table 6: Immunohistochemistry Results and Patient Demographics of Prostate Samples

\begin{tabular}{|c|c|c|c|c|c|c|c|c|c|c|c|c|}
\hline Patient & $\begin{array}{c}\text { Age at } \\
\text { diagnosis }\end{array}$ & $\mathbf{O A}$ & $\begin{array}{r}\text { Survival } \\
\text { (months) }\end{array}$ & $\begin{array}{c}\text { Local } \\
\text { Recurrence }\end{array}$ & Metastasis & $\begin{array}{c}\text { Gleason } \\
\text { Score }\end{array}$ & $\begin{array}{c}\text { Initial } \\
\text { PSA (ng/ } \\
\text { mL) }\end{array}$ & CD31 & KI-67 & COMP & PSMA & Surgery \\
\hline 1 & 71 & - & 154.38 & No & No & $3+4$ & $\mathrm{n} / \mathrm{a}$ & $0.8 \%$ & $31.4 \%$ & $80.3 \%$ * & $2.8 \%$ & \\
\hline 2 & 77 & - & 87.5 & No & No & $4+5$ & 27.9 & $3.1 \%$ & $38.7 \%$ & $12.2 \%$ & $34.3 \%$ & \\
\hline 3 & 78 & - & 67.4 & No & No & $4+3$ & 4.9 & $0.4 \%$ & $0.6 \%$ & $0.7 \%$ & $3.1 \%$ & \\
\hline 4 & 74 & - & 74.9 & No & No & $4+4$ & 9.4 & $0.2 \%$ & $1.4 \%$ & $0.7 \%$ & $3.6 \%$ & \\
\hline 5 & 78 & - & 87.8 & No & No & $4+4$ & 5.5 & $0.6 \%$ & $2.4 \%$ & $2.3 \%$ & $17.1 \%$ & \\
\hline 6 & 60 & - & 49.7 & No & No & $3+4$ & 4.7 & $0.6 \%$ & $1.0 \%$ & $0.1 \%$ & $10.3 \%$ & \\
\hline 7 & 58 & - & 77.66 & No & No & $5+4$ & 11 & $0.4 \%$ & $3.8 \%$ & $7.3 \%$ & $4.3 \%$ & \\
\hline 8 & 69 & - & 44.84 & No & No & $4+3$ & 11.28 & $0.6 \%$ & $12.7 \%$ & $17.6 \%$ & $25.2 \%$ & \\
\hline 1 & 53 & + & 70.1 & No & Yes & $5+4$ & 15 & $1.0 \%$ & $3.8 \%$ & $1.9 \%$ & $12.4 \%$ & \\
\hline 2 & 72 & + & 101.55 & No & No & $3+4$ & 6.98 & $0.7 \%$ & $33.4 \%$ & $37.0 \%$ & $4.1 \%$ & \\
\hline 3 & 70 & + & 97.7 & No & No & $4+4$ & 91 & $2.6 \%$ & $72.7 \%$ & $21.1 \%$ & $39.2 \%$ & \\
\hline 4 & 74 & + & 76.22 & No & No & $3+5$ & 258.1 & $1.1 \%$ & $23.5 \%$ & $8.8 \%$ & $27.8 \%$ & \\
\hline 5 & 55 & + & 71.45 & No & Yes & $4+5$ & 14.6 & $0.8 \%$ & $0.8 \%$ & $8.7 \%$ & $24.7 \%$ & \\
\hline 6 & 65 & + & 45.43 & No & No & $3+4$ & 8.6 & $0.6 \%$ & $1.5 \%$ & $4.9 \%$ & $15.4 \%$ & $\begin{array}{l}\text { THA, } \\
\text { TKA }\end{array}$ \\
\hline 7 & 75 & + & 57.76 & No & No & $4+3$ & 6.51 & $1.7 \%$ & $18.1 \%$ & $31.7 \%$ & $28.1 \%$ & \\
\hline 8 & 61 & + & 57.66 & No & No & $4+5$ & 22.3 & $0.5 \%$ & $21.1 \%$ & $35.0 \%$ & $6.2 \%$ & \\
\hline 9 & 74 & + & 51.12 & No & No & $5+4$ & 13.01 & $1.4 \%$ & $40.6 \%$ & $51.3 \%$ & $5.2 \%$ & $\begin{array}{c}\text { Bi-TKA, } \\
\text { THA }\end{array}$ \\
\hline 10 & 60 & + & 43.22 & No & No & $4+3$ & 5.76 & $0.3 \%$ & $1.3 \%$ & $37.0 \%$ & $15.7 \%$ & TKA \\
\hline 11 & 70 & + & 43.49 & No & No & $4+5$ & 67.38 & $0.7 \%$ & $27.6 \%$ & $47.2 \%$ & $2.0 \%$ & Bi-THA \\
\hline 12 & 70 & + & 94.9 & No & No & $4+3$ & 4.2 & $0.8 \%$ & $1.7 \%$ & $2.0 \%$ & $2.4 \%$ & TKA \\
\hline
\end{tabular}

* Sample was excluded from analysis because of extreme outlier (>4SD from mean). OA: osteoarthritis; TKA: total knee arthroplasty. THA: total hip arthroplasty. Bi: bilateral.

of that in a patient without osteoarthritis is promising, novel and the first of its kind in the literature. This finding could pertain to our hypothesis and is therefore currently being studied at our institution. However, we understand the limitations of our sample size and as such regard this as preliminary information.

Some limitations are inherent to our studies. The retrospective nature of this analysis may have increased the potential for selection bias not only due to the fact that our institution is a tertiary referral center but because of exclusion of patients without possible predictors and/or lack of data on them. Moreover, there is the possibility of confounding because the patients in this study received a comprehensive prostate cancer treatment protocol which included radiation therapy, chemotherapy, and surgical resection. Furthermore, inherent characteristics to the each of the compared groups may alter the findings of any regression model. As such, variables included in the model allowed us to account for initial differences, such as Gleason score and hormonal therarpy, as has been performed in the past [26-28]. Mean age at death was also not evaluated and the effects of osteoarthritis on patients who died at a young age not studied. We were unable to account for age at onset of osteoarthritis or age at which osteoarthritis was diagnosed, which may influence disease progression over time. An a priori power analysis was also not performed as no previous studies had evaluated the effect of osteoarthritis on prostate cancer and as such no estimation of an effect size could be made. The number of patients who underwent joint arthroplasty was limited; however, the relationship between arthroplasty and prostate cancer disease progression is novel and evaluations of larger patient cohorts are needed. Future studies will aim to determine whether high levels of COMP in the prostate or the serum and/or whether the severity of osteoarthritis impacts its relationship with prostate cancer.

In conclusion, this study identified a relationship between osteoarthritis and the development of metastatic disease in patients with prostate cancer. Patients with osteoarthritis expressed higher COMP levels in the prostate tissue and most likely in distant lymphatic nodes. Moreover, our findings suggest that joint arthroplasty may mitigate the effect of osteoarthritis on metastasis, which 
Table 7: Immunohistochemistry Results and Patient Demographics of Lymph Node Samples

\begin{tabular}{|c|c|c|c|c|c|c|c|c|c|c|}
\hline Patient & Age & Status & $\mathbf{O A}$ & $\begin{array}{c}\text { \% Positive } \\
\text { PSMA }\end{array}$ & $\begin{array}{c}\% \text { Positive } \\
\text { COMP }\end{array}$ & $\begin{array}{c}\text { \% Positive } \\
\text { KI-67 }\end{array}$ & $\begin{array}{c}\text { Gleason } \\
\text { Score }\end{array}$ & $\begin{array}{c}\text { Initial PSA } \\
(\mathbf{n g} / \mathbf{m L})\end{array}$ & $\begin{array}{c}\text { Local } \\
\text { recurrence }\end{array}$ & Metastasis \\
\hline 1 & 74 & alive & - & $0.27 \%$ & $7.06 \%$ & $2.79 \%$ & $3+3$ & 4.48 & 0 & 0 \\
\hline 2 & 76 & alive & - & $0.45 \%$ & $27.08 \%$ & $13.43 \%$ & $3+3$ & 6.1 & 0 & 0 \\
\hline 4 & 81 & alive & - & $1.80 \%$ & $30.71 \%$ & $2.63 \%$ & $4+4$ & 17.5 & 0 & 0 \\
\hline 5 & 65 & alive & + & $0.67 \%$ & $61.39 \%$ & $7.59 \%$ & $5+4$ & 15 & 0 & 1 \\
\hline 6 & 71 & alive & - & $2.38 \%$ & $16.35 \%$ & $3.63 \%$ & stage 3 & $\mathrm{n} / \mathrm{a}$ & 1 & 1 \\
\hline 7 & 73 & dead & - & $7.48 \%$ & $22.60 \%$ & $7.84 \%$ & stage 4 & $\mathrm{n} / \mathrm{a}$ & 1 & 1 \\
\hline
\end{tabular}

OA: osteoarthritis

could impact treatment protocols and survival outcomes of the second most common cause of cancer-related deaths in men in the United States.

\section{MATERIALS AND METHODS}

A retrospective case-control study was performed by reviewing the clinical data of all prostate cancer patients treated at a tertiary referral center from 2000 to 2016. The study was approved by the Wake Forest University Health Sciences Institutional Review Board. Inclusion criteria comprised patients treated for prostate cancer at our institution over the age of 18 , disease stage and follow up time over 6 months. Exclusion criteria were patients under the age of 18, and those with a tumor different from primary prostate cancer. Patients were stratified by concomitant diagnosis of osteoarthritis retrieved from the patients' medical record and absence of osteoarthritis or joint diseases different from osteoarthritis (including rheumatoid arthritis) (Figure 3).

Data extracted included patient demographics, osteoarthritis presence or absence, Gleason score, history of joint surgery, type of joint surgery, use of radiation therapy, chemotherapy use, prostatectomy, hormonal therapy, age at diagnosis, progression free survival, and outcomes including local recurrence, distant metastasis, and death. Patient surgical history and imaging was assessed for total joint arthroplasty within the study period. Local recurrence was defined as clinical, radiographic or pathologic evidence of disease at the primary site or prostate bed. Distant metastasis was defined as any evidence of disease outside the primary and regional lymph nodal basins. Progression-free survival was defined as the duration of time to any disease recurrence or death. Time-to-event outcomes were calculated from the time of diagnosis to the event.

Immunohistochemistry (IHC) was used to evaluate prostate cancer samples and lymph nodes for a variety of markers with COMP being the main outcome of interest. Briefly, the cancer patient database was queried for tissue samples of patients included in this study. A total of 20 samples were identified and ordered at random by the Core Pathology Department at Wake Forest University.
Full cohort evaluation was not possible due to the low availability of samples.

The prostate core biopsies and open biopsies were fixed with formalin and embedded in paraffin before sectioning. A total of four, $5 \mu \mathrm{m}$ slice samples were obtained for each of the 20 patients. The lymph nodes were obtained and prepared in a similar fashion. IHC protocols were performed following the manufacturer's protocols. Hematoxylin and Eosin (H\&E) staining was done as per our laboratories protocol. Pathology reports were extracted for the H\&E stains to determine Gleason score per trained pathologists. IHC for angiogenesis using CD31 (Abcam, Cambridge, UK, Antibody ab28364 at 1:300 dilution with specificity for human, mouse and porcine tissue), COMP (Abcam, Cambridge, UK, Antibody ab231977 at 1:500 dilution with specificity for human and mouse samples), proliferation using Ki-67 (MilliporeSigma, Burlington, MA, Antibody ab9260 at 1:500 dilution with specificity for human, mouse and rat), and PSMA (prostate specific membrane antigen, Dako Agilent Technologies, Santa Clara, CA, m3620 at 1:70 dilution, reactive to human samples) were performed. A blinded reviewer calculated the percentage of positively stained cells by IHC within the tissues utilizing the VisioPharm Software (Hoersholm, Denmark) as previously described [29].

Statistical analysis was comprised of descriptive statistics, logistic regressions, and Cox regression models. The linearity of the continuous variables with respect to the logit of the dependent variable was assessed via the Box-Tidwell procedure. A Bonferroni correction was applied during assumption evaluation of the models. Initial unadjusted comparisons were also performed and presented herein. Model adjustments were made based on covariates including age at diagnosis, presence or absence of osteoarthritis, hormonal therapy, chemotherapy, Gleason score, and joint arthroplasty. Three different Cox models were performed to evaluate time to local recurrence, time to metastasis and time to death. Comparison of groups at baseline was done to test for possible differences and for determining if there was a need for propensity score matching. Chi-square tests and Mann-Whitney tests were used as appropriate following evaluation of data normality 
for the latter test. Given that the groups were clinically similar, as well as their treatment, it was deemed that propensity score matching was not necessary. Survival was estimated using the Kaplan-Meier method for the cohort of patients in which histology was performed. No statistical analysis was performed on the lymphatic node samples of IHC given the low sample size $(n=7)$, as to avoid both type 1 and type 2 errors. On IHC analysis samples were excluded if their expression was 4 standard deviations above or below the mean (in total one sample was excluded).

\section{Abbreviations}

COMP: Cartilage Oligomeric Matrix Protein; ECM: Extracellular Matrix (ECM); PSMA: Prostate Specific Membrane Antigen; IHC: Immunohistochemistry ; PSA: Prostate Specific Antigen; THA: Total Hip Arthroplasty; TKA: Total Knee Arthroplasty.

\section{Author contributions}

Samuel Rosas designed the experiment collected, analyzed, and interpreted the data, and co-wrote the manuscript.

Ryan Hughes collected, analyzed, and interpreted the data, and co-wrote the manuscript

Michael Farris designed the experiment, collected, analyzed, and interpreted the data, and co-wrote the manuscript.

Hwajin Lee: collected, analyzed, and interpreted the data.

Emory R. McTyre: assisted with the design of the study, and collected, analyzed, and interpreted the data.

Johannes F. Plate analyzed, and interpreted the data, and co-wrote the manuscript.

Lihong Shi: analyzed, and interpreted the data, and co-wrote the manuscript.

Cynthia L. Emory co-wrote the manuscript.

A. William Blackstock acquired funding for the study and assisted with conception of the study.

Bethany A. Kerr PhD conceived and designed the experiment, collected, analyzed, and interpreted the data, and co-wrote the manuscript.

Jeffrey S. Willey PhD conceived and designed the experiment, collected, analyzed, and interpreted the data, and co-wrote the manuscript, and acquired funding.

\section{ACKNOWLEDGMENTS}

We would like to thank Andy Kwok, BS, Joseph Moore, BS, the Core Imaging Laboratory and the Tumor Tissue Pathology Shared Resource Center at Wake Forest School of Medicine for their assistance. We would also like to thank Drs. E. Ann Tallant, and Patricia Gallagher for help and support.

\section{CONFLICTS OF INTEREST}

The authors have no conflicts to disclose.

\section{FUNDING}

This work was supported in part by: The National Institute of Arthritis and Musculoskeletal and Skin Diseases of the National Institutes of Health under R21AR072806; the Chronic Disease Research Fund at the Wake Forest School of Medicine under the direction of the Hypertension and Vascular Research Group, and the Cardiovascular Sciences Center. This work was supported by the Wake Forest Baptist Compressive Cancer Center's Tumor Tissue \& Pathology Shared Resource supported by the National Cancer Institute's Cancer Center Support Grant award number P30CA012197. An author of this article was partially supported by a T-32 grant from the National Institutes of Health to the Department of General Surgery at Wake Forest School of Medicine T32 GM099606/GM/NIGMS; another author was partially supported by The Dubie Holliman Heart and Cancer Fund.

\section{REFERENCES}

1. Siegel RL, Miller KD, Jemal A. Cancer statistics, 2019. 2019; 69: 7-34. https://doi.org/10.3322/caac.21551. [PubMed].

2. Christie DR, Sharpley CF. How Accurately Can Prostate Gland Imaging Measure the Prostate Gland Volume? Results of a Systematic Review. Prostate Cancer. 2019; 2019:6932572. h https://doi.org/10.1155/2019/6932572. [PubMed].

3. James ND, Sydes MR, Clarke NW, Mason MD, Dearnaley DP, Spears MR, Ritchie AW, Parker CC, Russell JM, Attard G, de Bono J, Cross W, Jones RJ, et al and STAMPEDE investigators. Addition of docetaxel, zoledronic acid, or both to first-line long-term hormone therapy in prostate cancer (STAMPEDE): survival results from an adaptive, multiarm, multistage, platform randomised controlled trial. Lancet. 2016; 387: 1163-77. https://doi.org/10.1016/ S0140-6736(15)01037-5.

4. Parker CC, James ND, Brawley CD, Clarke NW, Hoyle AP, Ali A, Ritchie AW, Attard G, Chowdhury S, Cross W, Dearnaley DP, Gillessen S, Gilson C, et al and Systemic Therapy for Advanced or Metastatic Prostate cancer: Evaluation of Drug Efficacy (STAMPEDE) investigators. Lancet. 2018; 392: 2353-66. https://doi.org/10.1016/ S0140-6736(18)32486-3.

5. Roehrborn CG, Black LK. The economic burden of prostate cancer. BJU Int. 2011; 108: 806-13. https://doi.org/10.1111/ j.1464-410X.2011.10365.x. [PubMed].

6. Trogdon JG, Falchook AD, Basak R, Carpenter WR, Chen RC. Total Medicare Costs Associated With Diagnosis and Treatment of Prostate Cancer in Elderly Men. JAMA Oncol. 2019; 5: 60-66. https://doi.org/10.1001/ jamaoncol.2018.3701. [PubMed].

7. Peisch SF, Van Blarigan EL, Chan JM, Stampfer MJ, Kenfield SA. Prostate cancer progression and mortality: a review of diet and lifestyle factors. World J Urol. 2017; 35: 867-74. https://doi.org/10.1007/s00345-016-1914-3. [PubMed]. 
8. Cerami E, Gao J, Dogrusoz U, Gross BE, Sumer SO, Aksoy BA, Jacobsen A, Byrne CJ, Heuer ML, Larsson E, Antipin Y, Reva B, Goldberg AP, et al. The cBio cancer genomics portal: an open platform for exploring multidimensional cancer genomics data. Cancer Discov. 2012; 2: 401-4. https://doi.org/10.1158/2159-8290.CD-12-0095. [PubMed].

9. Englund E, Canesin G, Papadakos KS, Vishnu N, Persson E, Reitsma B, Anand A, Jacobsson L, Helczynski L, Mulder H, Bjartell A, Blom AM. Cartilage oligomeric matrix protein promotes prostate cancer progression by enhancing invasion and disrupting intracellular calcium homeostasis. Oncotarget. 2017; 8: 98298-311. https://doi.org/10.18632/ oncotarget.21176. [PubMed].

10. Harris KS, Kerr BA. Prostate Cancer Stem Cell Markers Drive Progression, Therapeutic Resistance, and Bone Metastasis. Stem Cells Int. 2017; 2017: 8629234. https:// doi.org/10.1155/2017/8629234. [PubMed].

11. Tseng S, Reddi AH, Di Cesare PE. Cartilage Oligomeric Matrix Protein (COMP): A Biomarker of Arthritis. Biomark Insights. 2009; 4: 33-44. https://doi.org/10.4137/BMI.S645.

12. Englund E, Bartoschek M, Reitsma B, Jacobsson L, Escudero-Esparza A, Orimo A, Leandersson K, Hagerling C, Aspberg A, Storm P, Okroj M, Mulder H, Jirström K, et al. Cartilage oligomeric matrix protein contributes to the development and metastasis of breast cancer. 2016; 35: 5585-96. https://doi.org/10.1038/onc.2016.98. [PubMed].

13. Cisternas MG, Murphy L, Sacks JJ, Solomon DH, Pasta DJ, Helmick CG. Alternative Methods for Defining Osteoarthritis and the Impact on Estimating Prevalence in a US Population-Based Survey. Arthritis Care Res (Hoboken). 2016; 68: 574-80. https://doi.org/10.1002/ acr.22721. [PubMed].

14. Nayak P, Luo R, Elting L, Zhao H, Suarez-Almazor ME. Impact of Rheumatoid Arthritis on the Mortality of Elderly Patients Who Develop Cancer: A Population-Based Study. Arthritis Care Res (Hoboken). 2017; 69: 75-83. https://doi. org/10.1002/acr.22997. [PubMed].

15. Wislowska M, Jablonska B. Serum cartilage oligomeric matrix protein (COMP) in rheumatoid arthritis and knee osteoarthritis. Clin Rheumatol. 2005; 24: 278-84. https:// doi.org/10.1007/s10067-004-1000-x. [PubMed].

16. Simon TA, Thompson A, Gandhi KK, Hochberg MC, Suissa $\mathrm{S}$. Incidence of malignancy in adult patients with rheumatoid arthritis: a meta-analysis. Arthritis Res Ther. 2015; 17: 212. https://doi.org/10.1186/s13075-015-0728-9. [PubMed].

17. Tangen CM, Faulkner JR, Crawford ED, Thompson IM, Hirano D, Eisenberger M, Hussain M. Ten-year survival in patients with metastatic prostate cancer. Clin Prostate Cancer. 2003; 2: 41-5. https://doi.org/10.3816/ CGC.2003.n.011. [PubMed].

18. Pascale M, Azinwi CN, Marongiu B, Pesce G, Stoffel F, Roggero E. The outcome of prostate cancer patients treated with curative intent strongly depends on survival after metastatic progression. BMC Cancer. 2017; 17: 651. https:// doi.org/10.1186/s12885-017-3617-6. [PubMed]
19. Kim MM, Hoffman KE, Levy LB, Frank SJ, Pugh TJ, Choi S, Nguyen QN, McGuire SE, Lee AK, Kuban DA. Improvement in prostate cancer survival over time: a 20-year analysis. Cancer J. 2012; 18: 1-8. https://doi. org/10.1097/PPO.0b013e3182467419. [PubMed].

20. Jeffers A, Sochat V, Kattan MW, Yu C, Melcon E, Yamoah K, Rebbeck TR, Whittemore AS. Predicting Prostate Cancer Recurrence After Radical Prostatectomy. Prostate. 2017; 77: 291-8. https://doi.org/10.1002/pros.23268. [PubMed].

21. Kim CK, Park BK, Lee HM. Prediction of locally recurrent prostate cancer after radiation therapy: incremental value of 3T diffusion-weighted MRI. J Magn Reson Imaging. 2009; 29: 391-7. https://doi.org/10.1002/jmri.21645. [PubMed].

22. Sridharan S, Macias V, Tangella K, Melamed J, Dube E, Kong MX, Kajdacsy-Balla A, Popescu G. Prediction of prostate cancer recurrence using quantitative phase imaging: Validation on a general population. Sci Rep. 2016; 6: 33818. https://doi.org/10.1038/srep33818. [PubMed].

23. Miyata Y, Mitsunari K, Asai A, Takehara K, Mochizuki Y, Sakai H. Pathological significance and prognostic role of microvessel density, evaluated using CD31, CD34, and $\mathrm{CD} 105$ in prostate cancer patients after radical prostatectomy with neoadjuvant therapy. Prostate. 2015; 75: 84-91. https://doi.org/10.1002/pros.22894. [PubMed].

24. Trojan L, Thomas D, Friedrich D, Grobholz R, Knoll T, Alken P, Michel MS. Expression of different vascular endothelial markers in prostate cancer and BPH tissue: an immunohistochemical and clinical evaluation. Anticancer Res. 2004; 24: 1651-6.

25. Verma R, Gupta V, Singh J, Verma M, Gupta G, Gupta S, Sen R, Ralli M. Significance of p53 and ki-67 expression in prostate cancer. Urol Ann. 2015; 7: 488-93. https://doi. org/10.4103/0974-7796.158507. [PubMed].

26. Wright JL, Salinas CA, Lin DW, Kolb S, Koopmeiners J, Feng Z, Stanford JL. Prostate cancer specific mortality and Gleason 7 disease differences in prostate cancer outcomes between cases with Gleason $4+3$ and Gleason $3+4$ tumors in a population based cohort. J Urol. 2009; 182: 2702-7. https://doi.org/10.1016/j.juro.2009.08.026. [PubMed].

27. Jo JK, Hong SK, Byun SS, Lee SE, Oh JJ. Comparison of clinical outcomes between upgraded pathologic Gleason score $3+4$ and non-upgraded $3+4$ prostate cancer among patients who are candidates for active surveillance. World J Urol. 2015; 33: 1729-34. https://doi.org/10.1007/s00345015-1527-2. [PubMed].

28. Huynh MA, Chen MH, Wu J, Braccioforte MH, Moran BJ, D'Amico AV. Gleason Score $3+5$ or $5+3$ versus $4+4$ Prostate Cancer: The Risk of Death. Eur Urol. 2016; 69: 9769. https://doi.org/10.1016/j.eururo.2015.08.054. [PubMed].

29. Kerr BA, Shi L, Jinnah AH, Willey JS, Lennon DP, Caplan AI, Byzova TV. Kindlin-3 Mutation in Mesenchymal Stem Cells Results in Enhanced Chondrogenesis. bioRxiv. 2019: 578690. https://doi.org/10.1101/578690. 